\title{
CROSSWORD PUZZLE
}

\section{How well do you know COVID-19?}

\section{B. M. Munasinghe}

Acting Consultant Anesthetist, District General Hospital, Mannar, Sri Lanka.

ORCID- 0000-0001-8373-4752; Meethenwala, Walakadawaththa, Mawathagama, Sri Lanka; E-mail: malakafmp@gmail.com; Phone: +94773645827; Mobile: 94773645827

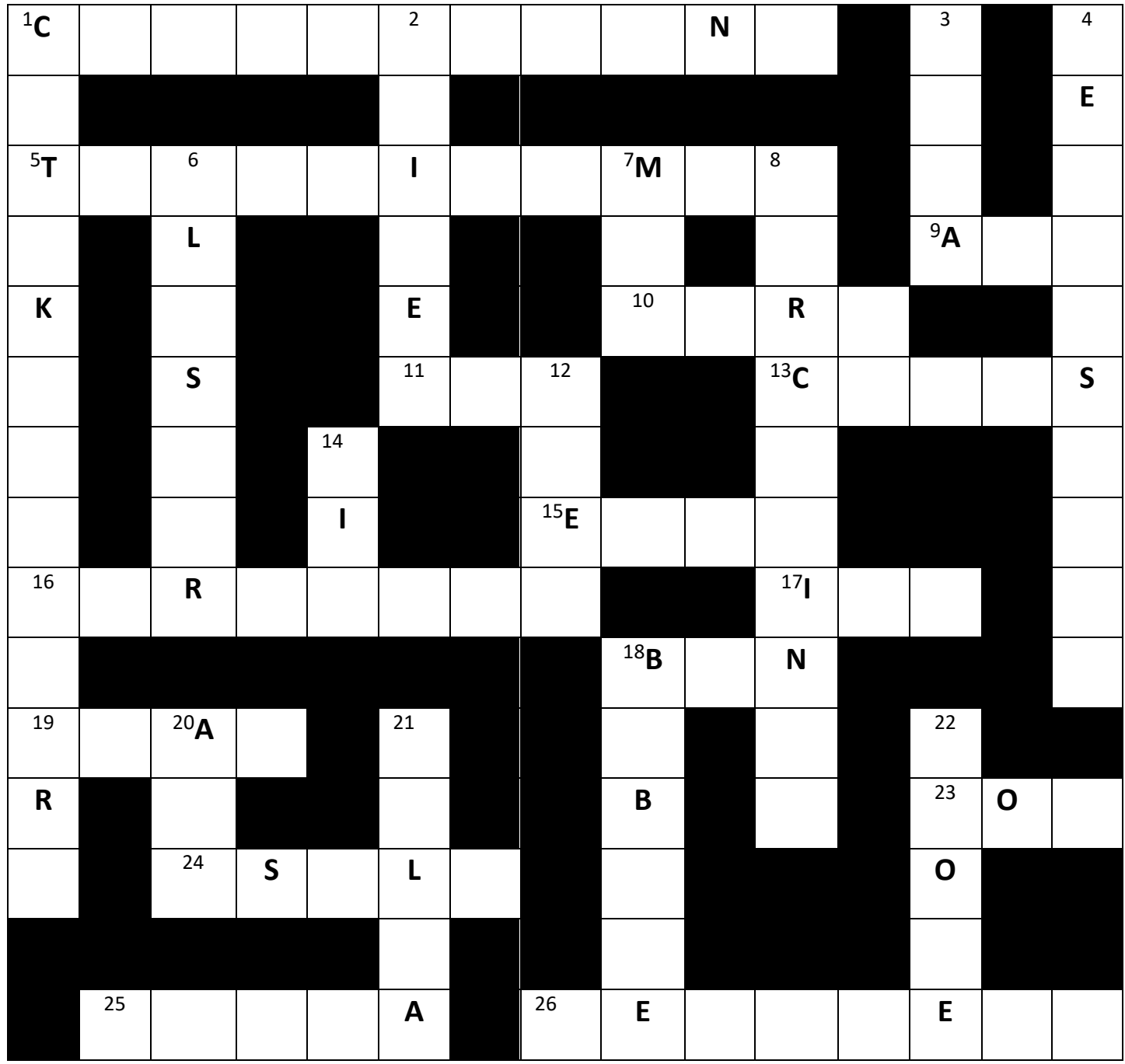

\section{ACROSS}

1. Defined as thickened interlobular septa and intralobular lines superimposed on diffuse ground-glass opacities, a radiological feature of progressive COVID-19 disease but non-specific.

5. A humanized monoclonal antibody used as a therapy in selected moderate to severe COVID-19 group, recently widely discussed in local media due to scarcity and cost

9. Recommended by the European Resuscitation Council to be used in indicated, out-of-hospital cardiac arrests of suspected or confirmed COVID-19 patients, short form. 
10. Defined as "an acute respiratory illness with a history of fever or measured fever of $\geq 38^{\circ} \mathrm{C}$ and cough, with onset within the past 10 days, requiring hospitalization", Increases COVID-19 related morbidity and mortality, short form 11. COVID-19 belongs to this group of viruses. Short form

13. Formation of these in the vasculature are increasingly seen among COVID-19 patients needing risk stratification and treatment.

15. These strategies are utilized during the COVID-19 pandemic in-order to maintain the case load, return to near normal public life and achieve economic recovery.

16. The virus implicated in COVID-19

17. A cytokine released as a response in multitude of inflammatory and infectious conditions including COVID-19, Targeted by (5)

18. The country of origin of COVID-19 had to ......... trading and consuming wild animals as food due to the likely source of COVID-19. Hint: verb

19. An uncommon route of acquiring COVID-19.

23. An index utilized in predicting risk of intubation in COVID-19 patients. Short form

24. A type of a pulmonary adverse effect due to self-generation of large tidal volumes in affected COVID-19 patients, not specific to COVID-19. Short form

25. The country where the index case of COVID-19 was reported.

26. The clinical trial which demonstrated the prognostic benefits of dexamethasone therapy in COVID-19

\section{DOWN}

1. An aggressive inflammatory response with release of a large amount of pro-inflammatory cytokines seen in COVID-19, which leads to adverse outcomes

2. A vaccine given in two doses for COVID-19 patients, identified by the generic name, BNT162b2

3. These filters are used in negative-pressure rooms during the COVID-19 pandemic to extract $99 \%$ of pathogens of 0.3 microns. Short form

4. Intravenous nucleotide pro-drug which inhibits replication of COVID-19 pathogen, used with dexamethasone

6. Concentration of infection at the same place at the same time. Hint: noun

7. A syndrome which is not specific but reported in increased numbers, especially among children with COVID-19. Severe form associated with elevated inflammatory markers and severe multisystem involvement. Short form

8. A Janus kinase inhibitor used in COVID-19. Combined therapy with (4) has shown to benefit patients receiving high flow therapy or non-invasive ventilation

12. COVID-19 virus enters cells via this receptor. Short form

14. An uncommon type of coagulopathy seen among COVID-19 patients. Short form

18. A concept where mask use or physical distancing with close contacts are not practiced.

20. These procedures for COVID-19 patients need full personal protective equipment worn by the health care workers. Short form

21. A variant of COVID-19 virus which is largely responsible for the third wave in Sri Lanka

22. This position has shown to improve oxygenation in both awake and intubated COVID-19 patients. 